\title{
画HAD
}

DOI: http://doi.org/10.22585/hospdomic.v1i4.22

\section{Estudio comparativo entre pacientes EPOC ingresados en hospitalización a domicilio y hospitalización convencional.}

\section{Comparative study between COPD patients admited in hospital at home and conventional hospitalization}

Pablo Ruiz-Sada', María-José Esquillor-Rodrigo², Lara Palacios-García ${ }^{3}$, María-Jesús Iguzquiza-Pellejero', Idoia Oroz-Segura ${ }^{4}$, Isabel Resano-García ${ }^{4}$

1. MD, Servicio Medicina Interna, Hospital Reina Sofía, Tudela (Navarra), España.

2. PhD, Servicio Medicina Interna, Hospital Reina Sofía, Tudela (Navarra), España.

3. MD, Servicio de Medicina Interna, Hospital Universitario de Basurto, Bilbao, España.

4. DUE, Servicio Medicina Interna, Hospital Reina Sofía, Tudela (Navarra), España.

Correspondencia/Correspondence

Pablo Ruiz-Sada.

Servicio de Medicina Interna, Hospital Reina

Sofía, Tudela (Navarra), España.

Calle Donantes de Sangre número 19. CP

31523.

pablitasitas@gmail.com

Recibido/Received

23.06.2017

Aceptado/Accepted

06.10 .2017

\section{Conflicto de Intereses/Competing interest}

Ninguno que declarar

CÓMO CITAR ESTE TRABAJO | HOW TO CITE THIS PAPER

Ruiz-Sada P, Esquillor-Rodrigo M J, Palacios-García L, Iguzquiza-Pellejero M J, Oroz-Segura I, ResanoGarcía I. Estudio comparativo entre pacientes EPOC ingresados en hospitalización a domicilio y hospitalización convencional. Hosp Domic. 2017;1 (4): 189-97 


\section{RESUMEN}

Objetivo: Demostrar el riesgo añadido derivado de la Hospitalización Convencional ( $\mathrm{HC}$ ) cuando ésta puede ser sustituida por la Hospitalización a Domicilio (HAD) en el contexto de reagudizaciones de Enfermedad Pulmonar Obstructiva Crónica (EPOC).

Pacientes y métodos: Estudio de cohortes histórico con análisis retrospectivo y comparativo de datos en que se seleccionan pacientes ingresados en HAD desde el 2010 hasta la actualidad. Se comparan 30 pacientes con reagudización de EPOC que cumplían criterios de ingreso en $\mathrm{HAD}$ e ingresan en HAD con 30 pacientes con reagudización de EPOC que cumplían criterio de HAD, pero ingresan en $\mathrm{HC}$.

Resultados: No se apreciaron diferencias en las características basales de los dos grupos. Se midieron los reingresos en el hospital y la mortalidad por reagudización de EPOC y por otras causas al mes del alta. En el grupo HC se observó mayor reingreso por reagudización de EPOC 0,333 (IC 0,118-0,943), mayor reingreso global 0,345 (IC 0,155-0,772) así como mayor mortalidad global 0,103 (IC 0,15-0,696).

Conclusiones: Nuestro estudio sugiere que existe un riesgo aumentado de reingreso y mortalidad al mes atribuible a la $\mathrm{HC}$ tras un episodio de reagudización de EPOC.

Palabras clave: Servicios de Atención a Domicilio Provisto por Hospital; Enfermedad Pulmonar Obstructiva Crónica; Readmisión del Paciente; Mortalidad.

\section{ABSTRACT}

Aim: To demonstrate the risk derived from Conventional Hospitalization $(\mathrm{CH})$ when it can be replaced by Hospital at Home $(\mathrm{HAH})$ units in the context of Chronic Obstructive Pulmonary Disease exacerbations.

Patients and methods: Historical cohort study with retrospective and comparative data analysis in which patients admitted to HAD are selected from 2010 to the present. 30 patients with exacerbation of who met criteria for admission to $\mathrm{HAH}$ and entered $\mathrm{HAH}$, compared with 30 patients who met $\mathrm{HAH}$ criterion but who entered $\mathrm{CH}$.

Results: There were no differences in the baseline characteristics of the two groups. Hospital readmissions and mortality due to exacerbation of COPD and other causes were measured one month after discharge. In the $\mathrm{CH}$ group, greater re-admission due to exacerbation of COPD was observed .333 (Cl 0.118-0.943), greater global readmission $0.345(\mathrm{Cl} 0.155-0.772)$ and greater overall mortality 0.103 (Cl 0.15-0.696).

Conclusions: Our study suggests that there is an increased risk of readmission and mortality after one month attributable to $\mathrm{HC}$ after an episode of exacerbation of COPD.

Keywords: Home Care Services, Hospital-Based; Pulmonary Disease, Chronic Obstructive; Patient Readmission; Mortality 


\section{INTRODUCCIÓN}

La Enfermedad Pulmonar Obstructiva crónica (EPOC) supone una obstrucción crónica y poco reversible al flujo aéreo asociada principalmente al humo del tabaco $(1,2)$. Causa una gran morbilidad y mortalidad a escala global, provocando todos los años la muerte de al menos 2,9 millones de personas. Actualmente es la cuarta causa de muerte y será la tercera en 2020 (2). En España, 18.000 personas mueren al año de EPOC generando un gasto estimado en 909.5 euros al año por paciente (3). Teniendo en cuenta que el $80 \%$ deriva de gastos hospitalarios y fármacos, la Hospitalización a Domicilio (HAD) se ha erigido como una alternativa a la Hospitalización Convencional (HC) en un intento de disminuir los costes generados por la enfermedad.

Más allá de la mejoría de parámetros económicos derivados de la implantación de las Unidades de HAD para pacientes con EPOC (Estudio Delphi), muchos expertos consideran la HAD como beneficiosa para el manejo de los pacientes con agudizaciones de EPOC al evitar la posibilidad de contraer infecciones nosocomiales, mejorar la efectividad de tratamientos como la oxigenoterapia, la ventilación mecánica, la rehabilitación en domicilio y potenciar la adherencia a tratamientos crónicos.

Existen numerosos datos hoy en día que indican que pacientes seleccionados podrían ser tratados en el domicilio. Desde estudios no controlados (4) como controlados y aleatorizados (5). En dichos trabajos, se consigue la recuperación del paciente sin un aumento en la tasa de reingresos, recaídas o fracasos terapéuticos. No existe, sin embargo, ningún estudio que al comparar la HC con la HAD demuestre un riesgo de reingreso o mortalidad atribuible a la HC. El propósito de este estudio es comparar la HC frente a la HAD en el contexto de reagudización EPOC.

\section{MATERIAL Y MÉTODOS}

Se diseñó un estudio de cohortes históricas para analizar de forma retrospectiva 30 pacientes diagnosticados de reagudización de EPOC que ingresaron en HD y comprarlos con 30 que ingresaron el HC. El estudio fue aprobado por el Comité Ético de Investigación Clínica local. Se seleccionan pacientes con EPOC según la clasificación GOLD (6) ingresados en HAD desde el 2010 hasta la actualidad.

El diagnóstico de reagudización de EPOC se realizó en base a la historia clínica, exploración física, datos de laboratorio y radiológicos. El aumento de disnea basal, el aumento del volumen y/o purulencia del esputo y la fiebre fueron los datos clínicos escogidos para definir clínicamente reagudización de EPOC. Los parámetros a destacar en la exploración física fueron la presencia de taquipnea, $\mathrm{SpO} 2<90 \%$ y la auscultación pulmonar de roncus o sibilancias. La elevación de reactantes de fase aguda, el descenso de pO2 <60 mmHg y la elevación de Pco2 $>50 \mathrm{~mm} \mathrm{Hg} \mathrm{lo}$ fueron en la analítica. En cuanto a la radiología valoramos datos indirectos de EPOC y de afectación parenquimatosa pulmonar aguda.

El abordaje estándar inicial siguió las recomendaciones de GesEPOC (7). Durante su estancia en urgencias, el paciente fue valorado por el equipo de medicina interna que ofreció, si procedía, el ingreso en HAD. Previa firma de consentimiento informado, se incluyeron pacientes con neumonía, como sin ella, que cumplían criterios de inclusión en HAD, siempre en función de disponibilidad de camas (Tabla 1). Consecuentemente, tomamos dichos criterios de inclusión y exclusión como nuestros para el estudio. Añadimos, sin embargo, como criterio de exclusión para nuestro análisis, el retorno de pacientes procedentes de HAD a la HC por la razón que fuese y el fallecimiento durante el ingreso a estudio. 


\begin{tabular}{|l|}
\hline Tabla 1. Criterios de inclusión y exclusión en HAD \\
\hline $\begin{array}{l}\text { Estabilidad clínica } \\
\text { Respiratoria: SpO2 aceptable para flujos máximos de } 3 \text { litros/minuto } \\
\text { Hemodinámica: TAM asumible para aportes máximos de } 1 \mathrm{~L} / 24 \text { horas }\end{array}$ \\
\hline Diagnóstico definido \\
\hline Pertenecientes al área sanitaria \\
\hline Domicilio condiciones salubridad \\
\hline Cuidador 24 horas \\
\hline Teléfono de contacto \\
\hline
\end{tabular}

El grupo de HC ingresó en planta y siguió el mismo protocolo terapéutico que el grupo de HAD bajo la estrategia de trabajo habitual en la planta de Medicina Interna. Los pacientes ingresados en HAD se trasladaron en ambulancia al domicilio y recibieron visita de médico internista y enfermera el día siguiente. Ambos grupos se dirigieron a un régimen u otro tras ser valorados por el equipo médico en urgencias. Permanecieron en dicho servicio el tiempo necesario para la estabilización clínica.

Tras la valoración inicial, el médico estableció el plan terapéutico y el circuito a seguir para demandar asistencia sanitaria urgente y programada. A partir de ahí, se mantuvo una tónica de visitas diarias por enfermería y cada 48 horas por el médico, excluyendo los fines de semana, siempre y cuando el curso clínico no requiriera modificar la estrategia. Haciendo honor a la patología de base bronquial presente en estos pacientes, todos son citados en consultas de Medicina Interna 1 mes tras el alta.

Se recogieron variables cuantitativas continuas como la edad, la saturación de oxígeno, la tensión arterial sistólica y diastólica, la frecuencia cardíaca, el valor de urea y creatinina al diagnóstico, el Índice Barthel, número de ingresos previos por EPOC y los días de estancia. Como variables cualitativas se recogieron el sexo, el hecho de ser "reagudizador" ( $>2$ reagudizaciones el año previo), llevar oxígeno domiciliario, estar institucionalizado, presentar deterioro cognitivo (Test Pfeiffer $>3$ errores), la presencia de factores de riesgo (consumo de tabaco y alcohol) y comorbilidades (HTA, DM, hepatopatía, insuficiencia cardíaca, anemia y neoplasia), presentar eventos en el ingreso destacables (síndrome confusional, diarrea, desnutrición) así como el reingreso y mortalidad acumulados al mes del episodio por EPOC, por otra causa o en global. Estas últimas seis variables fueron las variables principales.

Se compararon las características basales de los grupos utilizándose $\mathrm{CHI}$ cuadrado y la prueba de la T. Se observaron los eventos en relación a la presencia o no del factor de riesgo: Hospitalización convencional. Se calcularon Riesgos Relativos (RR) de reingreso y mortalidad asociados a la Hospitalización Convencional. Debido al reducido tamaño muestral no se pudo realizar análisis multivariante. Se intentó, sin embargo, relacionar los hallazgos con las variables medidas. Se revisaron las Historias clínicas electrónicas de los pacientes y se construyó una base de datos con el programa SPSS versión 23.0. 


\section{RESULTADOS}

Se compararon todas las variables entre el grupo Hospitalización Convencional (Grupo de HC) y el grupo Hospitalización Domiciliaria (Grupo de HD) sin observarse diferencias significativas en ninguna. La "p" es NS, mayor a 0,05 concluyéndose que son grupos comparables. Las características de los sujetos incluidos se muestran en la Tabla 2. Se apreció, sin embargo mayor dispersión en la variable "Días de estancia" en el Grupo de HD (Figura 1). En dicho grupo el paciente que menor tiempo estuvo ingresado permaneció solo 8 días y el que más 17, lo que contrasta con el mínimo y el máximo del Grupo HC, 6-12 respectivamente.

\begin{tabular}{|c|c|c|}
\hline & HAD SI & HAD NO \\
\hline EDAD & $81,33 \mathrm{DE} 10,172$ & $80,4 \mathrm{DE}$ 9,91 \\
\hline SEXO & $80 \%$ MUJERES $20 \%$ HOMBRES & $60 \%$ MUJERES 40\% HOMBRES \\
\hline ESTADÍO GOLD & $\begin{array}{l}\text { 1: } 13,3 \% \\
2: 20 \% \\
\text { 3: } 36,7 \% \\
4: 30 \%\end{array}$ & $\begin{array}{l}\text { 1: } 16,7 \% \\
\text { 2: } 23,3 \% \\
\text { 3: } 20 \% \\
\text { 4: } 40 \%\end{array}$ \\
\hline ESTACIÓN & $\begin{array}{l}\text { Invierno } 40 \% \\
\text { Resto } 60 \%\end{array}$ & $\begin{array}{l}\text { Invierno } 46 \% \\
\text { Resto } 54 \%\end{array}$ \\
\hline REAGUDIZADOR & $60 \%$ & $50 \%$ \\
\hline OXÍGENO DOMICILIARIO & $53,3 \%$ & $40 \%$ \\
\hline INSUFICIENCIA CARDÍACA & $56,7 \%$ & $63,3 \%$ \\
\hline HTA & $73 \%$ & $80 \%$ \\
\hline DM & $80 \%$ & $43 \%$ \\
\hline HEPATOPATÍA & $3,3 \%$ & $3,3 \%$ \\
\hline ALCOHOL & $23,3 \%$ & $36,7 \%$ \\
\hline TABACO & $20 \%$ & $36,7 \%$ \\
\hline ANEMIA & $16,7 \%$ & $13,3 \%$ \\
\hline NEOPLASIA & $16,7 \%$ & $10 \%$ \\
\hline DETERIORO COGNITIVO & $33,3 \%$ & $26,7 \%$ \\
\hline
\end{tabular}




\begin{tabular}{|l|l|l|}
\hline \multicolumn{2}{|l|}{ Tabla 2. Características basales sujetos incluidos } & $20 \%$ \\
\hline INSTITUCIONALIZADO & $10 \%$ & $30 \%$ \\
\hline FIEBRE & $20 \%$ & $26,7 \%$ \\
\hline RETENCIÓN DE CARBÓNICO & $26,7 \%$ & $26,7 \%$ \\
\hline CONFUSIONAL AL INGRESO & $33,3 \%$ & $6,7 \%$ \\
\hline DIARREA AL INGRESO & $10 \%$ & $30 \%$ \\
\hline DESNUTRICIÓN AL INGRESO & $20 \%$ & 11,05 DE 8,61 \\
\hline DÍAS DE ESTANCIA & 13,27 DE 6,49 & $36,7 \%$ \\
\hline BARTHEL < 35 & $26,6 \%$ & $30 \%$ \\
\hline
\end{tabular}

Figura 1. Dispersión variable días de estancia
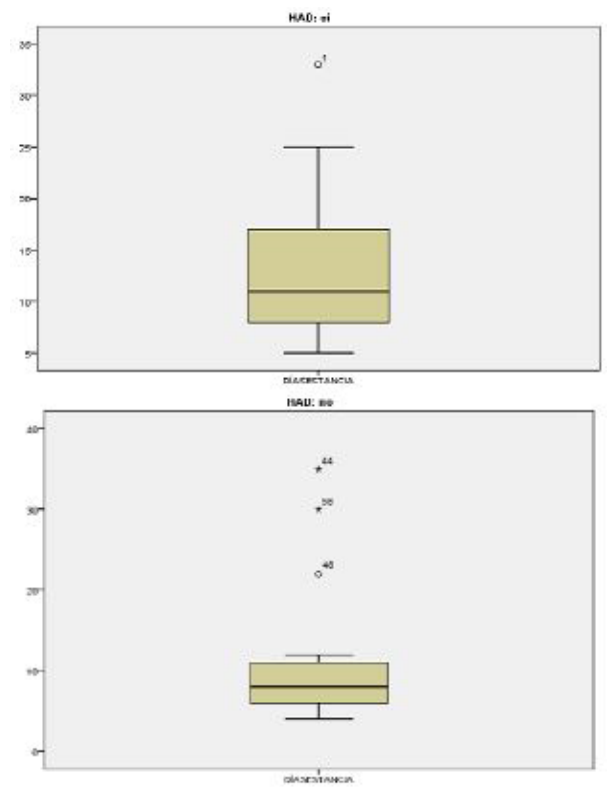

Se calculó RR de reingreso por reagudización de EPOC 0,333 (IC 0,118-0,943), de reingreso por otra causa 0,722 (IC 0,284-1,834), de mortalidad por reagudización de EPOC 0,154 (0,023-1,01), por otra causa 0,37 (IC 0,65-2,29), reingreso global 0,345 (IC 0,155-0,772) y mortalidad global 0,103 
(IC 0,15-0,696). Como se aprecia, los cálculos son estadísticamente significativos para el reingreso por EPOC, para el reingreso global y la mortalidad global (Tabla 3). Expresado de otra manera (1/ RR), encontramos estadísticamente significativo que la hospitalización convencional en nuestro estudio implica una probabilidad 3 veces mayor de reingreso por EPOC en el primer mes, 2,93 veces mayor de reingreso global y 9,7 veces de mortalidad global.

\begin{tabular}{|l|l|l|}
\hline \multicolumn{3}{|c|}{ Tabla 3. Detalle de la N y el \% de las variables resultado del estudio } \\
\hline & Grupo de HD & Grupo de HC \\
\hline & N\% & N \% \\
\hline Reingreso por EPOC & 516,66 & 1343,33 \\
\hline Reingreso por otra causa & 620 & 413,33 \\
\hline Reingresos globales & 1136,66 & 1756,66 \\
\hline Mortalidad por EPOC & 00 & 930 \\
\hline Mortalidad por otra causa & 13,33 & 26,66 \\
\hline Mortalidad total & 13,33 & 1136,66 \\
\hline
\end{tabular}

No se realizó análisis multivariante por el escaso número de pacientes incluidos en el estudio. Sin embargo, se realizó análisis comparativo de las variables con el reingreso o la mortalidad derivadas del episodio. Así, no se encontró diferencias en reingreso ni en mortalidad en función de la estación del año, ni en función de ser reagudizador o estar en tratamiento con oxígeno domiciliario. Se observa, sin embargo, cómo el 20\% del grupo de estadíos GOLD bajo (1 y 2) mueren frente al $30 \%$ del grupo estadíos avanzados.

\section{DISCUSIÓN}

La EPOC es un proceso de alta prevalencia, cuyas reagudizaciones provocan muchas hospitalizaciones con alto impacto socioeconómico. La experiencia ha demostrado que un tratamiento y seguimiento continuado por parte de una unidad de HAD permite estabilizar al paciente con una eficacia similar a la hospitalización convencional (8-9).

Un alto porcentaje de los ingresos en las unidades de HAD son por EPOC. En nuestra unidad, suponen el $7,5 \%$ del total (10). Cuando se pone a disposición del paciente técnicas y procedimientos diagnostico-terapéuticos que permiten su tratamiento integral en domicilio, disminuyen las complicaciones derivadas del ingreso convencional, y aumenta su calidad de vida.

Múltiples estudios prospectivos aleatorios $(4,5)$ demuestran que el tratamiento del EPOC en HAD aumenta la calidad de vida y disminuye los costes sanitarios. Con todos estos datos la sociedad de HAD, la SEMERGEN (9) y la propia Sociedad Europea de respiratorio, recomiendan en sus do- 
cumentos de consenso el manejo en el domicilio de las reagudizaciones moderadas de la EPOC, reservando el hospital para las reagudizaciones graves (11).

La robustez del estudio radica en que los grupos son comparables. Se confrontan todas las variables sin observarse diferencias significativas entre ellas. Aun así, no se recogieron datos clínicos y biológicos al ingreso y durante el mismo como la necesidad de corticoides endovenosos, de broncodilatadores nebulizados, de ventilación mecánica no invasiva o la presencia de gérmenes resistentes. Sólo conociendo una distribución homogénea de estos datos entre los dos grupos se podría concluir que el mayor porcentaje de reingresos el grupo $\mathrm{HC}$ es atribuible a la propia $\mathrm{HC}$.

El riesgo de reingreso asociado al episodio en el grupo de $\mathrm{HC}$ es prácticamente tres veces mayor, presentando, además, mayor mortalidad. Estos resultados deben ser interpretados con cautela. Podrían ser debidos, a aspectos desfavorables de la HC (infecciones nosocomiales, síndrome confusional-agitación, factores dietéticos o desnutrición) que fueron medidos como objeto del estudio y se distribuyeron por igual entre los dos grupos. Podrían, sin embargo, también deberse a otras variables confusoras, que no han sido medidas. Por ejemplo, se desconoce la existencia de cuidador en casa de los pacientes ingresados en $\mathrm{HC}$ o no, cosa que influiría en una mayor tasa de reingresos tras el alta.

En cuanto a las causas concretas que justifican estos resultados las desconocemos. En principio no ha sido el propósito del presente estudio y trabajos con un mayor número de pacientes son necesarios para ello en el futuro.

Así pues, se puede concluir que el estudio sugiere que existe un riesgo aumentado de reingreso y mortalidad al mes atribuible a la HC tras un episodio de reagudización de EPOC. Trabajos con más pacientes en un futuro son necesarios para determinar el por qué.

\section{BIBLIOGRAFÍA}

1. Balaguer C, Carrera M, Agustí A. Enfermedad pulmonar obstructiva crónica (I). Medicine. 2006;9(63):4077-9. DOI: 10.1016/S0211-3449(06)74381-8

2. Laredo LM. Fármacos en el tratamiento de la enfermedad obstructiva crónica. En: Lorenzo P, Morena A, Loza JC, Lizardian I, Moro MA, editores. Farmacología básica y clínica. $17^{a}$ edición. Madrid, España: Editorial Panamericana; 2005. p.907-18.

3. Masa JF, Sobradillo V, Villasante C, Jimenez Ruiz CA, Fernández Fau L, Viejo JL, Miravitlles M. Costes de la EPOC en España. Estimación a partir de un estudio epidemiológico poblacional. Arch Bronconeumol. 2004;40(2):72-9. DOI: 10.1016/S0300-2896(04)75476-9; PMID: 14746730

4. Gravil JH, Al-Rawas OA, Cotton MM, Flanigan U, Irwin A, Stevenson RD. Home treatment of exacerbations of chronic pulmonary disease by an acute respiratory assesment service. Lancet. 1998;351(9119): 1853-5.

5. Ram S, Wedzicha JA, Wright J, Greenstone M. Hospital at home for patients with acute exacerbations of chronic obstructive pulmonary disease: systematic review of evidence. BMJ Br Med J. 2004;329(7461):315. DOI: 10.1136/bmj.38159.650347.55; PMID: 15242868

6. Global Strategy for the Diagnosis, Management and Prevention of COPD, Global Initiative for Chronic Obstructive Lung Disease (GOLD) 2017 [Website]. Bethesda, USA: National Heart, Lung, and Blood Institute \& US National Institutes of Health \& World Health Organization; 2016 [accessed August 17, 2017]. Available from: http://goldcopd.org 
7. Miravitlles M, Soler-Cataluña JJ, Calle M, Molina J, Almagro P, Quintano JA, et al. Guía española de la EPOC (GesEPOC). Actualización 2014. Arch Bronconeumol. 2014;50(Supl 1):1-16. DOI: 10.1016/S0300-2896(14)70070-5; PMID: 24507959

8. Left B, Burton L, Mader SL, Naughton B, Burl J, Inouyr SK et al. Hospital at home: Feasibility and outcomes of a program to provide hospital-level care for acutely older patients. Ann Intern Med. 2005;143(11):798-808. DOI: 10.7326/0003-4819-143-11-200512060-00008; PMID: 16330791

9. Arnedillo Muñoz A. Consenso sobre atención integral de las agudizaciones de la enfermedad pulmonar obstructiva crónica (ATINA-EPOC): Parte V. Semergen. 2013;39(1):41-7. DOI: 10.1016/j.semerg.2012.04.005; PMID: 23517896

10. Esquillor-Rodrigo MJ. Hospitalización a Domicilio. Una alternativa a la hospitalización tradicional [tesis de postgrado]. Zaragoza, España: Universidad de Zaragoza; 2016.

11. Siafakas NM, Vermiere P, Brie NB, et al. Optimal assessment and management of chronic obstructive pulmonary disease (COPD): The European Respiratory Society Task Force. Eur Resp J. 1995;8(8):1398-420. PMID: 7489808 